\title{
Vacuna anti hepatitis A
}

\author{
JOSÉ COFRÉ G. ${ }^{1}$ y KATIA ABARCA V. ${ }^{2}$
}

\section{Hepatitis A vaccine}

Inactivated hepatitis A vaccine is an valuable preventive prophylactic tool because of its high immunogenicity, capacity to induce immunological memory and high efficacy and safety profile. It may be utilized since 12 month age with excellent seroconversion. The several available hepatitis A vaccines are interchangeable facilitating its use in a public health setting. The best way to benefit countries like Chile with intermediate endemicity is to implement a cohort vaccination program and to use it in the control of circumscribed outbreaks. Moreover it represents an alternative strategy to human normal immunoglobulin for immunoprophylaxis in close contact at home and enclosed institutions. In this article we present an exhaustive review of published experiences that support these asseverations.

Key words: Hepatitis A, Vaccines; Inactivated; Immunogenicity; Efficacy; Safety; Public health; Expanded Program on Immunization

\section{Introducción}

La infección causada por virus de hepatitis A (VHA) es endémica en nuestro país con ciclos de mayor incidencia como se aprecia en un informe del Ministerio de Salud de Chile publicado en este mismo número ${ }^{1}$. Las posibilidades de control y eliminación del VHA son reales ya que el reservorio es exclusivamente humano, no se ha descrito la infección crónica y, si bien se han identificado 4 genotipos a lo largo del mundo, sólo se conoce un serotipo, de distribución universal, que está determinado por epítopes en las proteínas de superficie del virus, posiblemente VP1 y VP3, sin conocerse con precisión contra qué estructura se dirigen los anticuerpos neutralizantes ${ }^{2}$. En buenas cuentas el individuo infectado una vez no se reinfecta. Ésta es la premisa para diseñar vacunas y anunciar el control de la enfermedad, en la medida que las poblaciones sean inmunizadas artificialmente.

Este artículo presenta una actualización sobre las vacunas anti VHA preparadas con el virus inactivado, sus propiedades inmunológicas, y el impacto clínico-epidemiológico de su aplicación. No se abordan los antecedentes relativos a vacunas atenuadas cuya experiencia y documentación científica se han centrado fundamentalmente en China.

\section{Composición}

Las cepas contenidas en las vacunas inactivadas contra VHA han sido aisladas de deposiciones de pacientes y adaptadas a cultivos celulares. Todas han sido traspasadas finalmente múltiples veces en fibroblasto de pulmón fetal humano MRC-5 desde donde son cosechadas, inactivadas con formalina y tituladas mediante unidades, no equivalentes entre sí, de manera que la potencia de los productos de uso clínico se expresa en forma no comparable entre las diferentes marcas (Tabla 1). Ninguna contiene antimicrobianos. Los productos Havrix ${ }^{\circledR}$ (belga), Avaxim ${ }^{\circledR}$ (francés) y Vaqta ${ }^{\circledR}$ (norteamericano) contienen hidróxido de aluminio como coadyuvante inmunopotenciador. La vacuna Epaxal ${ }^{\circledR}$ elaborada por el Instituto Suizo de Sueros y comercializada por Berna, Biotech Ltda., ha sido desarrollada con la estrategia de vehiculizar el VHA en la superficie de virosomas; esta estrategia se denomina IRIV (immunopotentiating reconstituted influenza virosomes). Para ello se ha empleado liposomas de fosfatidilcolina y fosfatidiletanolamina con la

\footnotetext{
Unidad de Infectología, Servicio de Pediatría, Hospital Luis Calvo Mackenna.

2 Unidad de Infectología, Servicio de Pediatría, Hospital Clínico Pontificia Universidad Católica de Chile
} 
adición de la hemaglutinina y neuraminidasa del virus influenza cepa A/Singapore/6/68/H1N1 cuya función es facilitar la presentación del virión de hepatitis $\mathrm{A}$ al sistema inmune y su rápido procesamiento ${ }^{3}$.

\section{Vacunas existentes en el mercado internacional: (Tabla 1)}

Con la introducción de Havrix junior ${ }^{\circledR}$ se discontinuó la comercialización de Havrix ® 360 UE cambiándose el esquema inicial de aplicación $(0,1,6$ meses $)$ por el simplificado $(0,6-12$ meses).

\section{Vacunas licenciadas en Chile:}

Avaxim ${ }^{\circledR}$, Epaxal ${ }^{\circledR}$, Havrix ${ }^{\circledR}$ y Havrix junior®. En trámite Avaxim ${ }^{\circledR} 80$ UI.

\section{Disponibilidad en el mercado nacional:}

Avaxim ${ }^{\circledR}$ (160 UI), Havrix ${ }^{\circledR}$ (1.440 UE)/ Havrix junior® (720 UE). En trámite de internación Epaxal®.

\section{Inmunogenicidad}

Las características que se describen a continuación son comunes para los cuatro productos existentes en el mercado internacional; si bien se han observado diversidad en los títulos alcanzados al comparar dos vacunas en un mismo ensayo, se estima que estas diferencias son clínicamente irrelevantes porque todos los títulos son muy superiores a los estimados protectores, son del orden de $10^{3}-10^{4} \mathrm{mUI} / \mathrm{ml}^{2}$.

Respuesta inmune a vacunas anti hepatitis A y criterio de protección. La administración de inmunoglobulina normal por vía intramuscular tiene alta eficacia protectora $(\sim 85 \%)$ si se aplica una dosis de $0,02 \mathrm{mk} / \mathrm{kg}$ de peso en las dos primeras semanas desde producido el contacto con la fuente de infección ${ }^{4}$. En estas condiciones se alcanzan concentraciones de anticuerpos neutralizantes en sangre de 10 a 20 mUI/ $\mathrm{ml}$, tras uno a dos meses de aplicar la inmunoglobulina normal, de lo que se ha extrapolado que concentraciones de 10, 20 ó $33 \mathrm{mUI} / \mathrm{ml}$ de anticuerpos anti VHA post vacunación, represen$\tan$ fielmente un valor de corte que predice protección contra la infección ${ }^{2}$.

Respuesta inmune primaria. Los títulos de anticuerpos inducidos por las vacunas son inferiores a los conferidos por la infección natural (rangos $10^{3}-10^{4}$ versus $10^{5}-10^{6}$ respectivamente $)^{2}$. No obstante, el 85 a $95 \%$ de los adultos inmunocompetentes seronegativos (75 a $80 \%$ en personas sobre 40 años) que reciben la primera dosis de vacuna, presentan anticuerpos anti VHA en el plazo de 10 a 14 días, sobre los niveles protectores $^{5-8}$; a los 28 días cerca del $100 \%$ alcanzó títulos protectores, y en una concentración superior a la primera determinación ${ }^{5-8}$. Esta respuesta es similar en niños de toda edad ${ }^{9-12}$, con títulos algo menores si la vacuna es administrada en el primer año de vida ${ }^{13-18}$. En estudios comparativos se ha observado mayores títulos de anticuerpos otorgados por algunas vacunas que otras, lo que no parece tener mayor relevancia en el corto ni a largo plazo pues se trata de diferencia entre concentraciones que son siempre 10 a 100 veces superiores a las protectoras.

Memoria inmunológica anti VHA. Existe comprobación experimental de que individuos vacunados con preparados inactivados anti VHA desarrollan inmunidad celular (LT) específica temprana $^{19,20}$ y tras 6 años de aplicada la vacuna, la respuesta inmune anti VHA mediada por células persiste $^{21}$, sugiriendo fuertemente que existen poblaciones de LT con memoria específica anti hepatitis A.

Respecto de la inmunidad humoral, inmediatamente antes de administrar la segunda dosis de vacuna se observa una caída de los títulos de anticuerpos anti VHA aunque permanecen por

Tabla 1. Esquemas de aplicación. vacunas inactivadas antihepatitis A

\begin{tabular}{lcccc}
\hline Nombre comercial & Avaxim ${ }^{\circledR}$ & Epaxal ${ }^{\circledR}$ & Havrix & Vaqta \\
\hline Fabricante & Aventis & Swiss Serum Institute & GlaxoSmith-Kline & Merck \& Cia \\
Cepa & GBM & RG - SB & HM 175 & CR326F \\
Cultivo de elaboración & MRC-5* & MRC-5* & MRC-5* & MRC-5* \\
Carga antigénica & $80 \mathrm{U}$ & $24 \mathrm{UI}$ & $360 \mathrm{UE}$ & $25 \mathrm{UI}$ \\
& $160 \mathrm{U}$ & & $720-1.440 \mathrm{UE}$ & $50 \mathrm{UI}$ \\
Dosificación (meses) & $0,6-12$ & 0,12 & $0,1,6-12$ & $0,6-18$ \\
& & & $0,6-12$ & \\
\hline
\end{tabular}

* ver texto. 
sobre el límite protector. Una segunda dosis a 618 meses de la primera, produce una respuesta inmune segundaria (booster): más rápida, más intensa, y mediada por IgG, en $100 \%$ de los individuos, independientemente de los títulos residuales pre booster y de la edad del sujeto ${ }^{21-23}$.

Duración de la inmunidad con esquema completo. La primera de las vacunas inactivadas aplicadas en humanos tiene tan sólo 12 años de uso clínico, de manera que la duración última de la inmunidad que globalmente confieren estas vacunas, es desconocida aún. Sin embargo, el seguimiento de individuos vacunados sin exposición posterior a la infección natural (adultos viajeros provenientes de países con muy bajo riesgo de infección) y en individuos que sí viven en un ambiente de endemia, ha permitido extrapolar conclusiones sobre esta materia. En la Tabla 2 se presentan los resultados observados o estimados con modelos matemáticos sobre la duración de la inmunidad con un esquema completo de vacunación.

Más allá de la comprobación de cuánto duran los títulos de anticuerpos protectores después de un esquema "completo" de vacunación o de su estimación matemática, la inducción de memoria inmunológica por una parte, y el booster natural dado por la circulación del virus en regiones endémicas de hepatitis $\mathrm{A}^{12}$, permiten predecir que es estas regiones habrá una protección a muy largo plazo con las vacunas anti VHA y que el riesgo de desplazar la infección hacia grupos de mayor edad si se aplica vacuna en edades tempranas, no será tal.
Edad mínima de vacunación. Los ensayos de campo diseñados para medir la eficacia de estas vacunas en Tailandia ${ }^{32}$, Nueva York $^{33}$ y Nicaragua $^{12}$, documentaron la seguridad y protección otorgada por estas vacunas sobre 2 años de vida; no hubo representación suficiente de niños bajo 24 meses de edad de manera que la Food and Drug Administration -FDA y su contrapartida europea, la European Medicine Evaluation Agency -EMEA licenciaron estas vacunas a partir de los 2 años de edad. Sin embargo, estudios de inmunogenicidad posteriores efectuados en lactantes en su segundo año de vida con Avaxim 80® UI y Havrix ${ }^{\circledR} 720 \mathrm{UE}^{34,35}$, comprobaron que ya a esa edad la inmunidad materna no interfiere sobre la frecuencia ni la intensidad de la respuesta inmune específica $y$, considerando estos nuevos antecedentes, se autorizó la licencia en Europa y algunos países latinoamericanos, a partir de los 12 meses de edad. Bajo un año de vida, la transferencia pasiva de anticuerpos anti VHA sí interfirió parcialmente esta respuesta a vacuna Havrix ${ }^{\circledR} 720 \mathrm{UE}^{15}$, Havrix ${ }^{\circledR} 360$ UE $^{13,14}$ y Avaxim ${ }^{\circledR} 80$ UI $^{16-18}$ observándose menores títulos de anticuerpos anti VHA en hijos de mujeres seropositivas que en hijos de mujeres seronegativas, aunque la seropositividad de la madre no impidió la estimulación inmune y priming en el lactante por el antígeno vaccinal ${ }^{15,16}$.

Inmunidad de rebaño. La vacunación programática beneficia indirectamente a individuos de otras cohortes no vacunadas, hecho que fuera observado en Israel. En este país la vacunación de lactantes a los 18 y 24 meses de edad causó

Tabla 2

\begin{tabular}{|c|c|c|c|c|c|c|c|}
\hline Vacuna & Potencia & Esquema & $\begin{array}{l}\text { Población } \\
\text { estudiada }\end{array}$ & Seguimiento & $\begin{array}{c}\text { Sero } \\
\%\end{array}$ & $\begin{array}{c}\text { Duración } \\
\text { estimada de } \\
\text { inmunidad }\end{array}$ & Ref \\
\hline Havrix ${ }^{\circledR}$ & $760 \mathrm{UE}$ & $0-1-6 \mathrm{~m}$ & Adultos belgas & 3 años & 100 & $\begin{array}{l}53 \% \text { a } 10 \text { años } \\
34 \% \text { a } 20 \text { años }\end{array}$ & 24 \\
\hline Havrix $®$ & $720 \mathrm{UE}$ & $0-1-6 \mathrm{~m}$ & Adultos alemanes & 5 años & 100 & 10 años & 25 \\
\hline Havrix ${ }^{\circledR}$ & $720 \mathrm{UE}$ & $0-1-6 \mathrm{~m}$ & Adultos griegos & 5 años & 99 & 20 años & 26 \\
\hline Havrix $®$ & $1.440 \mathrm{UE}$ & $0-6 \mathrm{~m}$ & Adultos belgas & 6 años & 100 & $100 \%$ a $20-25$ años & 27 \\
\hline Havrix ${ }^{\circledR}$ & $720 \mathrm{UE}$ & $0-1-6 \mathrm{~m}$ & Niños canadienses & 5 años & 100 & $>25$ años & 28 \\
\hline Havrix $®$ & $360 \mathrm{UE}$ & $0-1-6 \mathrm{~m}$ & Niños chinos & 5 años & 100 & 25 años & 29 \\
\hline Avaxim $®$ & $160 \mathrm{UI}$ & $0-6 \mathrm{~m}$ & Adultos belgas & 3 años & 100 & $\begin{array}{l}53 \% \text { a } 10 \text { años } \\
34 \% \text { a } 20 \text { años }\end{array}$ & 24 \\
\hline Vaqta ${ }^{\circledR}$ & $25 \mathrm{UI}$ & $0-6$ a $18 \mathrm{~m}$ & Niños americanos & - & - & $50 \%$ a 20 años & 30 \\
\hline Epaxal® & $500 \mathrm{UR}$ & $0-12 \mathrm{~m}$ & Adultos suizos & 3 años & 100 & $95 \%$ a 20 años & 31 \\
\hline
\end{tabular}


una notable y rápida reducción de la incidencia de la enfermedad en 3 años, no sólo en las cohortes vacunadas sino que a toda edad ${ }^{36,37}$. En Butte County, California por otra parte, la vacunación de aproximadamente $2 / 3$ de los niños entre 2 y 12 años, produjo en 3 años una reducción de $94 \%$ en la incidencia de la enfermedad en todas las edades $^{38}$. Los individuos vacunados no sólo no enferman de hepatitis sino que además, si tienen contacto con el virus natural acusando este contacto con una nueva elevación de IgG específica anti VHA, no lo excretan en deposiciones; este fenómeno fue observado durante un ensayo de campo con Epaxal® en Nicaragua con un seguimiento exhaustivo de los niños vacunados ${ }^{12}$. En otro ensayo en Tailandia con la vacuna Havrix ${ }^{\circledR}$, se comprobó excreción por nuevo contacto con el virus pero por tiempos muy breves ${ }^{32}$.

No se conoce con exactitud aún con qué coberturas puede aspirarse a este beneficio indirecto de una vacunación grupal, aunque en experiencias de campo se ha observado que con coberturas de $80 \%$ se logró controlar un brote epidémico en Alaska, no siendo igual en aquellas áreas en que la cobertura alcanzó sólo a 50\% ${ }^{39}$.

Esquema de una dosis. Una dosis de cualquiera de las vacunas ya otorga anticuerpos en concentraciones protectoras, hecho que ha sido corroborado por el éxito de las campañas de vacunación efectuadas con una sola dosis ${ }^{38-45}$.

\section{Respuesta inmune a una segunda dosis de} vacuna diferida. Se ha efectuado seguimiento en adultos no expuestos a la infección natural (en Escandinavia) y que han recibido sólo la dosis inicial; en 25 adultos suecos que fueran vacunados antes de viajar a zonas endémicas, con una sola dosis de vacuna Havrix ® 1.440 UE y fueran revacunados a los 4-6 años, se comprobó que los títulos previos al booster eran negativos en 7 y habían descendido en 18/25 hasta una media geométrica de $32 \mathrm{mUI} / \mathrm{ml}$. Una segunda dosis de 1.440 UE estimuló en todos una respuesta secundaria lográndose altos títulos de anticuerpos específicos con una media geométrica de 2.993 $\mathrm{mUI} / \mathrm{ml}^{46}$. En igual forma, adultos suizos vacunados con Havrix® 1.440 UE y revacunados 24 a 66 meses más tarde (promedio de 35 meses) con igual dosis, tuvieron respuesta tipo secundaria con títulos similares a quienes recibieran un esquema de vacunación convencional: 3.342 (95\% IC 2.618-4.266) versus 3.258 (95\% IC 2.5654.140) $\mathrm{mUI} / \mathrm{ml}$, respectivamente ${ }^{47}$. Con Epaxal ${ }^{\circledR}$ se ha repetido esta experiencia administrando a adultos una segunda dosis 18 a 54 meses más tarde que la primera y se observó alza muy significativa $(2.330-2.432 \mathrm{mUI} / \mathrm{ml})$ con esta segunda dosis ${ }^{48}$.

Efecto sinérgico: vacuna-booster natural. Con el seguimiento de 268 adultos y niños esquimales $(137<18$ años $)$ que recibieran una sola dosis de Havrix ${ }^{\circledR}$ - 1.440 UE sobre 20 años y 720 UE bajo esa edad-, y posiblemente continuaron expuestos al contacto con el virus natural, se comprobó que a 27 meses (rango 20-31) post vacunación, se conservaba aún inmunidad protectora y que la segunda dosis de vacuna evocó, tanto en niños como en adultos, una respuesta específica tipo memoria inmunológica ${ }^{49}$. Antes del booster la media geométrica de anticuerpos anti VHA era de $66 \mathrm{mUI} / \mathrm{ml}$ y sólo $16 \%$ tenían títulos bajo $20 \mathrm{mUI} / \mathrm{ml} \mathrm{(4 \%} \mathrm{entre} \mathrm{los} \mathrm{que} \mathrm{tenían} 0$ a 12 años al momento de recibir la primera dosis); después de este refuerzo se alcanzó un título en media geométrica de $1.544 \mathrm{mUI} / \mathrm{ml} \mathrm{y}$ $76 \%$ superaron los $750 \mathrm{mUI} / \mathrm{ml}(83 \%$ entre los que tenían 3 y 12 años al momento de la primera dosis).

Intercambiabilidad de vacunas. Estudios de aplicación de un esquema de dos dosis de vacuna empleando diferentes productos para la primera y la segunda inyección, han demostrado que la intensidad de la respuesta al booster es enteramente similar a la obtenida en individuos que recibieran las dos dosis del mismo producto. Esto ha sido evaluado para Vaqta ${ }^{\circledR}$ versus Havrix ${ }^{\circledR}$ en 201 adultos voluntarios en EUA, comparando las secuencias Havrix $® /$ Havrix $®$, Havrix $® /$ Vaqta ${ }^{\circledR}$, Vaqta ${ }^{\circledR} /$ Havrix ${ }^{\circledR}$, Vaqta ${ }^{\circledR} /$ Vaqta ${ }^{\circledR}$ en esquema 0-6 meses y seguimiento a 30 meses plazo $^{50}$. Las respuesta inmunes fueron comparables en título e intensidad en los cuatro grupos, al mes 85 a 94\% tenían seroconversión y a los 30 meses $100 \%$ había seroconvertido. Esta experiencia fue replicada en otro protocolo con 535 adultos norteamericanos que recibieran como primera dosis Havrix ${ }^{\circledR} 1.440 \mathrm{UE}$ y tras 24 a 52 meses de lapso, recibieran Vaqta ${ }^{\circledR} 50 \mathrm{U}$ o una segunda dosis de Vaqta ${ }^{\circledR} 1.440$ UE, llegando a similares conclusiones luego de un mes de seguimiento ${ }^{51}$. En otro ensayo, 185 adultos ingleses recibieron Havrix ${ }^{\circledR} 1.440$ UE como dosis inicial y alternativamente Havrix ${ }^{\circledR} 1.440$ o Avaxim 160 UI® como booster 6 a 7 meses más tarde; el seguimiento serológico tras un mes de la segunda dosis comprobó similar respuesta inmune en ambos grupos de estudio (media geométrica de 4.460 versus $6.669 \mathrm{mUI} / \mathrm{ml}$ respectivamente $)^{52}$. En otra experiencia con 117 adultos suizos viaje- 
ros que habían recibido una dosis de vacuna Havrix ${ }^{\circledR} 1.440$ UE 6 a 12 meses antes, se efectuó el booster con Epaxal® 500 UR, observándose un alza de anticuerpos anti VHA desde una media geométrica de $67 \mathrm{mUI} / \mathrm{ml}$ hasta 1.803 $\mathrm{mUI} / \mathrm{ml}^{53}$.

Esta documentada intercambiabilidad entre las diferentes vacunas facilitará los procesos de licitación pública que se aplican en los programas nacionales de vacunación ya que no se verá amenazada la protección de las cohortes de vacunados por eventuales cambios del origen del producto.

\section{Eficacia}

En tres ensayos controlados y doble ciego efectuados en niños, en zonas con alta endemia (Provincia de Kamphaeng Phet, Tailandia; Monroe, Nueva York; León, Nicaragua) se han establecido las siguientes eficacias de las vacunas anti VHA:

- En Tailandia, Havrix ${ }^{\circledR} 360$ UE, tuvo 94\% de eficacia (2/19.037 versus 32/19.120) contra infección sintomática con dos dosis (0-1 meses) en 33.174 niños entre 1 y 16 años $(0,1 \%$ era $<3$ años o $>14$ años) con un seguimiento de 11 meses. Se empleó como control la vacuna anti hepatitis B. La eficacia alcanzó a 100\% luego de administrar la tercera dosis 12 meses más tarde de la primera, con un plazo de observación aproximado de 500 días $^{32}$.

- En Nueva York, Vaqta® 25 UI, alcanzó 100\% de eficacia con una dosis (0/498 versus $25 /$ 496), en 519 niños entre 2 y 16 años, seguidos a 100 días plazo en promedio. Fue empleado como control un placebo. Se reportaron algunos casos en el grupo vacunado antes de 21 días de recibir la vacunación completa ${ }^{33}$.

- En Nicaragua, Epaxal® tuvo eficacia de $100 \%$ con una dosis de 24 UI (0/122 versus 17/117, en 122 niños entre 1,5 y 6 años de edad) con un seguimiento a 14 meses. Se usó un placebo como control. En el grupo vacunado se detectó 4 casos antes de 6 semanas de administrada la vacuna ${ }^{12}$.

\section{Seguridad}

Reactogenicidad. Las principales reacciones locales (dolor, irritación en el sitio de la inoculación) y generales (fiebre, rash, malestar general, cefalea) tienen una frecuencia similar a otras vacunas empleadas en el PAI ${ }^{34,54-56}$. En una evaluación preliminar, se aplicó la vacuna Havrix ${ }^{\circledR}$ a más de 500 niños argentinos entre 1 y 15 años observándose 10 a 15\% de alguna reacción local con las dos dosis de vacuna y refirieron dolor $\sim 20 \%$ de ellos ${ }^{34}$.

Las reacciones locales son atribuibles mayoritariamente a la presencia de $\mathrm{Al}(\mathrm{OH})^{3}$ en algunos de los productos, como se desprende al comparar la reactogenicidad entre los productos que incluyen este coadyuvante y la vacuna virosomal ${ }^{57}$. Estas vacunas anti VHA han sido elaboradas en cultivos de fibroblastos, líneas celulares debidamente libres de virus oncogénicos y que han sido empleadas por más de 20 años en la preparación de vacunas. Las escasas reacciones derivadas de la administración de estas vacunas no son consecuencia del empleo de estos cultivos celulares y la posibilidad que estas células diploides de origen humano contengan eventuales contaminantes biológicos es virtualmente descartable; garantía de ello son vacunas largamente utilizadas en humanos como polio inactivada y oral, rubéola, varicela y otras, preparadas en células diploides ${ }^{58}$.

\section{Efectos adversos mayores}

No se han reportado con vacuna Havrix ${ }^{\circledR}$ habiéndose evaluado sobre 120.000 dosis administradas ${ }^{56}$.

\section{Contraindicaciones}

Tratándose de un antígeno inactivado, la posibilidad de reversión a virulencia es inexistente, de manera que ni un estado de inmunocompromiso ni la gestación constituyen contraindicación para su aplicación. Si bien estas vacunas puedes ser administradas a pacientes inmunocomprometidos sin mayor riesgo para su salud, la respuesta inmune y la memoria inmunológica inducidas pueden ser inferiores a la observada en personas con su inmunidad indemne $e^{59,60}$.

\section{Indicaciones}

\section{- Uso de vacuna en el control de brotes epi- démicos.}

Hecha una revisión exhaustiva de la información disponible respecto a la eficacia de la vacuna en brotes epidémicos ${ }^{39,42,45,61-64}$, puede concluirse que la variabilidad en los resultados observados está condicionada por:

- tipo de brote epidémico del que se trate. Debe distinguirse brotes epidémicos en comunidades o centros cerrados con transmisión personapersona (centros de cuidado diario, internados, instituciones, familias); brotes epidémicos en comunidades pequeñas abiertas (villas, pueblos pequeños) y brotes epidémicos en grandes comunidades abiertas. 
- situación epidemiológica en que se inserte el brote epidémico. Así, no será igualmente efectiva una vacunación en una comunidad determinada si ella está inmersa en un área de baja endemia o en un área de mediana-alta endemia.

- oportunidad con que se realice la intervención, lo cual dependerá fundamentalmente de la capacidad del sistema de vigilancia para detectar precozmente la ocurrencia de un brote epidémico y la demora del sistema de salud para implementar la vacunación.

- cobertura de vacunación lograda en la población afectada.

La vacunación en brotes epidémicos en comunidades cerradas ha demostrado ser una medida eficaz. En un estudio randomizado en contactos intradomiciliarios de casos hospitalizados por hepatitis A, ocurrieron 2 casos de hepatitis en 173 vacunados y 12 casos en 178 no vacunados, con una eficacia protectora de $82 \%$ (IC 20-96) ${ }^{61}$. En un brote epidémico de hepatitis A en niños en un centro de refugiados en Croacia se vacunaron los 34 niños seronegativos, ocurriendo sólo un caso 5 días después de la vacunación y ningún nuevo caso posteriormente ${ }^{45}$.

La vacunación en brotes epidémicos en comunidades abiertas pequeñas o medianas en áreas no endémicas es también una medida eficaz, siempre que la intervención se realice en forma precoz y que la cobertura de vacunación sea alta. En varias comunidades rurales en Alaska que experimentaban frecuentes brotes epidémicos comunitarios, con la administración de una dosis de vacuna a cerca de 5.000 personas, se logró controlar el brote epidémico en 4 a 8 semanas en aquellas comunidades en que la cobertura de vacunación fue mayor al $80 \%$, mientras que en comunidades en que la cobertura no alcanzó al $50 \%$, la epidemia se mantuvo por más de 30 semanas ${ }^{39}$.

Varios son los ejemplos de uso de vacuna en brotes epidémicos que afectan a grandes comunidades abiertas, reportándose generalmente el control del mismo tras la vacunación. La administración de una dosis de vacuna a 500 y 4.500 niños en los barrios General Paz y comercial de Córdoba, Argentina, respectivamente, ha sido evaluada por investigadores argentinos como una medida altamente eficaz para el control de brotes comunitarios $^{62}$. En un brote epidémico de hepatitis ocurrido en dos villas en Eslovaquia, se observó una rápida declinación en el número de casos después de la vacunación de más de 600 niños en edad escolar ${ }^{42}$. En un extenso y prolongado brote epidémico de hepatitis A en Memphis, Tennessee, se implementó la vacunación de más de 30.000 niños observándose una declinación de casos en $64 \%$ durante el año siguiente ${ }^{63}$. Sin embargo, en esta situación los autores discutieron que el real impacto de la vacunación fue difícil de cuantificar considerando que la intervención se realizó después de varios meses de iniciado el brote epidémico, momento en que era esperable una declinación natural de los casos por agotamiento de los susceptibles.

Es posible esperar un efecto más notorio con una intervención más precoz y de alta cobertura, lo que en la práctica suele ser muy difícil de lograr. Un análisis sistemático de la literatura en este tópico concluyó que la evidencia disponible avala la eficacia de la vacuna en brotes epidémicos acaecidos en comunidades cerradas y en comunidades abiertas pequeñas en áreas no endémicas, siempre que la cobertura sea alta; pero que no se ha demostrado la eficacia de la vacunación en brotes epidémicos desarrollados en grandes comunidades abiertas en regiones endémicas ${ }^{64}$.

Aplicando esta información a la situación actual de nuestro país, se pueden detectar varios elementos que hacen predecir un bajo impacto del uso de vacuna en esta situación:

- demora en el reconocimiento de los brotes epidémicos y en la instauración de medidas de intervención,

- limitados recursos de personal que enfrente los brotes,

- limitada disponibilidad de vacuna y, sobre todo, que

- los brotes epidémicos, aun los institucionales o familiares, se están presentando inmersos en una situación epidémica que afecta a grandes comunidades abiertas.

\section{- Uso de la vacuna en forma de programa}

Existen en el mundo diversas experiencias en el uso de vacunación programática contra la hepatitis A. Las distintas estrategias subyacentes se pueden clasificar en:

- vacunación de determinados grupos etarios en zonas específicas de un país: ej. vacunación de adolescentes en Cataluña, España; lactantes mayores y adolescentes en la región de Puglia, Italia.

- estrategia in crescendo, con vacunación progresiva desde comunidades con mayor a menores tasas de hepatitis A: experiencia de EUA. - vacunación sistemática universal, nacional: experiencia de Israel.

Las experiencias de vacunación focalizada a ciertas poblaciones ha demostrado eficacia en reducir las tasas de hepatitis en las poblaciones 
vacunadas. Ejemplos de estas intervenciones son en primer lugar, la vacunación de adolescentes en Cataluña, España, donde a dos años de iniciado el programa se observó una reducción de las tasas globales de 6,2 a 2,6/100.000 hbtes y en el grupo vacunados de 10,3 a 1,8/100.000 hbtes ${ }^{65}$ $\mathrm{y}$, en segundo lugar, la vacunación de niños en el segundo año de vida y a los 12 años, iniciada en 1997 en la zona de Puglia, Italia ${ }^{66}$. Sin embargo, dado lo focalizado de estos programas, no han tenido impacto en la epidemiología nacional.

La experiencia de vacunación in crescendo ha sido implementada por E.U.A. En este país, el ACIP, recomendó en 1996 la vacunación a grupos de riesgo y a los niños de comunidades con tasas históricamente muy elevadas (nativos americanos y nativos esquimales) ${ }^{67}$. En 1999 amplió esta recomendación a los estados con las mayores tasas de hepatitis A del país (estados cuyas tasas duplicaban o más al promedio nacional en el decenio 1987-1997, esto era, tasas $\geq 20 / 100.000$ hbtes), e indicó considerar la vacunación en estados con tasas entre 10 y 20/100.000 hbtes ${ }^{68}$.

Como resultado de la primera etapa de esta estrategia, en que se logró una cobertura de 70\% con la primera dosis en las comunidades nativas, se ha observado una reducción desde tasas que llegaban a 100-450/100.000 hbtes durante los ciclos epidémicos, a tasas mantenidas bajo 10/ 100.000 hbtes, similares a las tasas del resto del país ${ }^{69}$. Como resultado de la segunda etapa se ha observado una reducción de $86 \%$ en estados donde la vacunación ha sido recomendada, de $89 \%$ en las áreas donde se indicó considerar la vacunación, y de $50 \%$ en los estados donde no se ha implementado una vacunación amplia ${ }^{69}$. Otro efecto de la estrategia descrita ha sido el aumento relativo de los casos de hepatitis en personas con edades mayores: de un predominio en el grupo etario de 5 a 9 años se ha pasado a un predominio a los 30-34 años. Cabe notar sin embargo, que se trata de un aumento relativo en relación al total de casos, y que las tasas específicas por edad se han reducido en todos los grupos etarios.

En un seguimiento realizado 9 años después de instaurada la vacunación en niños en la comunidad de Monroe, Nueva York, se ha observado un cambio de patrón epidemiológico, de frecuentes brotes epidémicos antes de la vacunación, a la desaparición sostenida de ellos, a pesar de la esporádica aparición de la infección en sujetos no vacunados ${ }^{33}$.

El único país del mundo que ha incorporado la vacunación contra la hepatitis A en forma sistemática y nacional es Israel. Allí conviven estrechamente comunidades beduinas con altas tasas de hepatitis y otras judías con tasas bajas; la incidencia promedio entre 1995 y 1998 fue de $41,5 / 100.000$ hbtes $(57,8 / 100.000$ en beduinos y $37,2 / 100.000$ en judíos). La vacunación fue incorporada en julio de 1999 al PAI a los 18 y 24 meses de edad, sin realizar vacunación sobre este límite (catch-up). La cobertura fue de 90 y $80 \%$ con la primera y segunda dosis respectivamente y la venta libre de vacuna a otras edades, representó el $10 \%$ del total de dosis. A 3 años de iniciada la vacunación (año 2002), se observó una disminución de las tasas a menos de 5/ 100.000 hbtes, esto es, una reducción de $90 \%$. La reducción de las tasas afectó a los dos grupos étnicos y no sólo a la cohorte vacunada, sino también a otro grupos etarios no vacunados ${ }^{36,37}$.

\section{- Uso en manejo de contactos en el hogar.}

Tradicionalmente se ha recomendado que, en los contactos de un paciente con hepatitis A al interior del hogar o en grupos con una similar dinámica de interrelación personal, se prescriba inmunoglobulina normal por su efecto universalmente protector, con una eficacia antes señalada de $\sim 85 \%$ dependiente de la dosis utilizada y el momento de colocación en relación con la exposición ${ }^{2}$; esta estrategia es de máximo rendimiento en la primera semana post exposición. Como su beneficio es transitorio y existe preocupación acerca de la seguridad biológica de los hemoderivados en general, se ha planteado la vacunación como alternativa más segura y que otorgaría inmunidad duradera. Al momento actual no se han comunicado experiencias comparativas de diseño que permitan cambiar esta conducta y la ACIP continúa recomendando en el RedBook 2003 sólo la prescripción de inmunogobulina ${ }^{70}$. Pese a la falta de evidencia científica, otros gru$\operatorname{pos}^{71}$ han optado por recomendar la vacunación si el contacto con un caso índice ha ocurrido en plazo menor a 7 días, o la vacunación más la inmunoglobulina normal simultánea pero aplicada en otro sitio del cuerpo, si han transcurrido hasta 14 días; más allá de este plazo, probablemente la vacunación no sea efectiva. Este criterio ha sido adoptado por los especialistas nacionales.

\section{Conclusiones}

De los antecedentes expuestos de desprende que:

- Las vacunas inactivadas anti hepatitis A son altamente inmunogénicas a partir del segundo año de vida. En consecuencia, más allá de la edad establecida en su registro sanitario en 
nuestro país, puede recomendarse su aplicación a partir de los 12 meses de edad.

- Las vacunas inactivadas anti hepatitis A estimulan el desarrollo de memoria inmune desde el primer año de vida; la inmunidad congénita trasplacentaria interfiere parcialmente en la intensidad de la respuesta humoral pero no inhibe su efecto priming específico.

- Con un esquema de dos dosis de vacuna inactivada, sin mediar un booster natural, se obtiene protección prolongada, ya comprobada a 6 años plazo y estimada en 20 o más años.

- En la evaluación de la inmunogenicidad de estas vacunas se ha reducido el número de dosis de vacuna (de 3 a 2), sin sacrificar la intensidad y frecuencia de la protección alcanzada.

- Si bien la mayor evaluación de la inmunogenicidad se ha desarrollado con dosis múltiples de estas vacunas, con una sola dosis se logra el priming en todas ellas.

- Una segunda dosis asegura la protección a virtualmente el $100 \%$ de las personas vacunadas y aumenta significativamente los títulos de anticuerpos circulantes.

- Las vacunas inactivadas anti hepatitis A existentes son intercambiables lo que facilita su aplicación programática e individual.

- La exposición a la infección natural por virus de hepatitis A en nuestro país continuará actuando como un refuerzo inmunitario para individuos que han recibido un esquema completo o monodosis de vacuna, mientras no se controle la infección por VHA.

- La eficacia comprobada en niños residentes en comunidades con alta endemia de infección oscila entre 94 y $100 \%$

- Una única dosis de vacuna es altamente inmunogénica y protectora para más del $90 \%$ de los vacunados, según se ha comprobado en el control de brotes epidémicos. En consecuencia, la mejor indicación para los contactos susceptibles de un caso índice, es administrarles una sola dosis de vacuna tempranamente.

- El manejo de brotes en comunidades cerradas debiera hacerse con vacuna y no con inmunoglobulinas ya que junto a su alta eficacia y seguridad, confiere una protección duradera a los vacunados.

- La vacunación programática otorga inmunidad de rebaño cuando las coberturas son elevadas en la población expuesta, probablemente sobre el $80 \%$.

- Los esquemas licenciados de vacunación apa- recen como rígidos; sin embargo, se dispone de suficientes antecedentes para aplicar elasticidad en su uso clínico individual. Los plazos establecidos de 6 a 12 ó 18 meses de lapso entre la primovacunación y la dosis booster pueden ser flexibilizados y ampliados, según se desprende de estudios de seguimiento serológico y de la comprobación de una memoria inmunológica anti VHA, sin perjudicar la calidad de la protección. Por razones operacionales, es aconsejable respetar el esquema que las autoridades sanitarias adopten en una vacunación programática.

- La reactogenicidad de las vacunas inactivadas anti hepatitis A es reducida y enteramente comparable a la producida por otras vacunas actualmente en uso en el PAI.

\section{Resumen}

La vacuna anti hepatitis A inactivada es una valiosa herramienta preventiva por su alta inmunogenicidad, inducción de memoria, eficacia y perfil de seguridad. Puede ser utilizada a partir de los 12 meses de vida con excelente seroconversión. Las diferentes vacunas son intercambiables entre sí facilitando su uso en salud pública. La mayor utilidad en países con endemia mediana como Chile está en su uso programático en cohortes y en el control de brotes epidémicos circunscritos. Además representa una estrategia alternativa a la inmunoglobulina normal en el manejo de contactos estrechos en el hogar y en instituciones cerradas. En este artículo se revisan in extenso las experiencias publicadas que fundamentan estas aseveraciones.

\section{Bibliografía}

1.- Medina B, Olea A, Aguilera X. Documento. Situación epidemiológica de la hepatitis A en Chile. Rev Chil Infect 2003; 20:

2.- Bell B P, Feinstone S M. Chapter 15: Hepatitis A Vaccine. En Vaccines, Plotkin SA and Orenstein WA, editors. 4th edition. WB Saunders Company, Philadelphia, 2003, 269-97.

3.- Moser C, Metcalfe I C, Viret J-F. Virosomal adjuvanted antigen delivery systems. Expert Res Vaccines 2003; 2 (2): 89-93.

4.- Kluge I. Gamma-globulin in the prevention of viral hepatitis: study of the effect of medium-size doses. Acta Med Scand 1963; 174: 469-77.

5.- Clemens R, Safary A, Hepburn A, Roche C, Stanbury W, André F. Clinical experience with an inactivated hepatitis A vaccine. J Infect Dis 1995; 171 (Suppl 10): S44-9.

6.- Ellebeck E, Lewis J, Nalin D et al. Safety profile and immunogenicity of an inactivated vaccine derived from an attenuated strain of hepatitis A. Vaccine 1992; 10: 668-71. 
7.- Garin D, Vidor E, Wallon M et al. Good immunogenicity of GBM strain inactivated hepatitis A vaccine in healthy male adults. Vaccine 1995; 13 (2): 220-4.

8.- Poovorawan Y, Theamboonlers, Chumdermpadetsuk S, Glück R, Cryz SJ Jr. Safety, immunogenicity and kinetics of the immune response to a single dose of virosome-formulated hepatitis A vaccine in Thais. Vaccine 1995; 13; 10: 891-3.

9.- Delem A, Safary A, De Namur F, Hauser P, D' Hondt P. Characterization of the immune response of volunteers vaccinated with a killed vaccine against hepatitis A. Vaccine 1993; 11: 479-84 .

10.- Lee S D, Lo K J, Chan C Y et al. Immunogenicity of inactivated hepatitis A vaccine in children. Gastroenterol 1993; 104: 1129-32.

11.- Block S, Hedrick J, Tyler R et al. Safety, tolerability, and immunogenicity of a formalin inactivated hepatitis A vaccine in children 4-12 years. In Program and Abstracts of the 31th Interscience Conference on Antimicrobial Agents and Chemotherapy, Chicago September 29-October 2, 1991. Washington DC. ASM Press 1991; abstract 314.

12.- Mayorga Pérez O, Herzog C H, Zellmeyer M, Loáisiga A, Frösner G, Egger M. Efficacy of virosome hepatitis A vaccine in young children in Nicaragua: Randomized placebo-control trial. J Infect Dis 2003 (1 September); 188; 671-7.

13.- Kanka G, Yalcin S S, Ceyhan M, Yurdakok K. Clinical trial to evaluate immunogenicity and safety of inactivated hepatitis A vaccination starting at 2month-old children. Turk J Pediatr 2000 Apr-Jun; 42(2): $105-8$.

14- Troisi C, Hollinger F B, Krause D S, Pickering L. Immunization of infants with hepatitis A vaccine. A comparative study of two dosing schedules. Proceedings of IX Triennal International Symposium on Viral Hepatitis and Liver Disease. Rome, Italy, 21-25 April 1996. Rizzetto M, Purcell RH, Gerin JL, Verme G, editors. Edizione Minerva Medica, Turin, 1997.

15.- Dagan R, Amir J, Mijalovsky A et al. Immunization against hepatitis $\mathrm{A}$ in the first year of life: priming despite the presence of maternal antibody. Pediatr Infect Dis J 200; 19 (11): 1045-52.

16.- Lagos R, Muñoz A, Dumas R et al. Immunological priming of one dose of inactivated hepatitis A vaccine given during the first year of life in presence of maternal antibodies. Vaccine 2003; 21: 3730-3

17.- Lieberman J M, Marcy M, Partridge S, Ward J I. Evaluation of hepatitis A vaccine in infants: effect of maternal antibodies on the antibody response. En Abstracts of the $36^{\text {th }}$ Annual Meeting of the Infectious Diseases Society of America, 1998, Denver, Alexandria; VA. Infectious Diseases of America. Abstr 76, p 76.

18.- Shapiro C N, Letson G W, Kuehn D. Effect of maternal antibody on immunogenicity of hepatitis A vaccine in infants. En Program and Abstracts of the $35^{\text {th }}$ Interscience Conference on Antimicrobial Agents and Chemotherapy, San Francisco, 17-20 September, 1995. ASM Press. Abstr H61.

19.- Cederna J B, Klinzman D, Stapleton J T. Inactivated hepatitis A vaccine elicits HAV-specific T cell proliferation. En Proceedings of IX Triennal International Symposium on Viral Hepatitis and Liver Disease. Rome, Italy, 21-25 April 1996; Rizzetto M, Purcell RH, Gerin JL, Verme G, editors. Edizione Minerva Medica, Turin, 1997. Abstr A78.

20.- Cederna J B, Klinzman D, Stapleton J T. Hepatitis A virus-specific humoral and cellular immune responses following immunization with a formalin-inactivated hepatitis A vaccine. Vaccine 2000: 18: 892-8.

21.- Van Damme P, Thoelen S, Cramm M. Inactivated hepatitis A vaccine: reactogenicity, immunogenicity and long-term antibody persistence. J Med Virol 1994; 44; 446-51.

22.- Vidor E, Salion P. Clinical development of a new inactivated hepatitis A vaccine. Sante 1998; 8 (5): 361-8.

23.- Ambrosch F, Wiedermann G, Jonas $\mathrm{S}$ et al. Immunogenicity and protectivity of a new liposomal hepatitis A vaccine. Vaccine 1997; 15 (11): 1209-13.

24.- Van Herck K, Beutels P, Van Damme P et al. Mathematical models for assessment of long-term persistence of antibodies after vaccination with two inactivated hepatitis A vaccines. J Med Virol 2000; 60: 1-7.

25.- Mainwald H, Jilg W, Bock H L, Löscher T, Sonnenburg F V. Long term persistence of anti HAV antibodies following active immunization with hepatitis A vaccine. Vaccine 1997; 15 (4): 346-8.

26.- Totos G, Gizaris V, Papaevangelou G. Hepatitis A vaccine: persistence of antibodies 5 years after the first vacination. Vaccine 1997; 15 (11): 1252-3.

27.- Van Herck K, Van Damme P. Inactivated hepatitis A vaccine-induced antibodies: Follow up and estimates of long term persistence. J Med Virol 2001; 63: 1-7.

28.- Van Damme P, Leroux-Roels G, Diaz-Mitoma F et al. Long term persistence of antibodies induced by vaccination and safety follow-up, with the first combined vaccine against hepatitis A and B in children and adults. J Med Virol 2001; 65: 6-13.

29.- Fan P, Chang M, Lee P, Safari A, Lee C H. Follow-up immunogenicity of an inactivated hepatitis A virus vaccine in healthy children: results after 5 years. Vaccine 1998; 16 (2-3): 232-5.

30.- Weins B, Bohidar N, Pigeon J, Egan J, Hurni W, Brown L, et al. Duration of protection from clinical hepatitis A disease after vaccination anti Vaqta. J Med Virol 1996; 49(3): 235-41.

31.- Bovier P A, Bock J, Farinelli T, Glück R, Herzog C. Long term immunogenicity of an inactivated virosome hepatitis A vaccine. J Med Virol 2002; 68 (4): 489-93.

32.- Innis B L, Snitbhan R, Kunasol P et al. Protection against hepatitis A by inactivated vaccine. JAMA 1994 May 4; 271 (17): 1328-34

33.- Werzberger A, Mensch B, Kuter B. A controlled trial of formalin-inactivated hepatitis A vaccine in healthy children. New Engl J Med 1992; 327: 453-7.

34.- López E L, Xifró M del C, Torrado L E et al. Safety and immunogenicity of a pediatric formulation of inactivated hepatitis A vaccine in Argentinean children. Pediatr Infect Dis J 2001; 20 (1): 48-52.

35.- Abarca K, Ibáñez I, Flores J, Vial PA, Safary A, Potin M. (Efficacy) Immunogenicity of hepatitis A vaccination in children aged 12 to 24 months. Arch Med Res 2001; 32 (5): 468-72

36.- Dagan R. Update from Israel, the first country to adopt hepatitis A universal immunization program. Libro de Resúmenes. X Congreso Mundial de Infectología Pediátrica, Santiago Chile, Noviembre 2002.

37.- Dagan R. Comunicación personal, agosto 2003. Datos no publicados.

38.- Averhoff F, Shapiro C N, Bell B P et al. Control of hepatitis A through routine vaccination of children. JAMA 2001; 286 (23): 2968-73.

39.- McMahon B J, Beller M, Williams J et al. A program to control an outbreak of hepatitis A in Alaska using 
inactivated hepatitis A vaccine. Arch Pediatr Adolesc Med 1996; 150 (7): 733-9.

40.- Thornburn K M. Immunization strategies to control a community-wide hepatitis A epidemic. Epidemiol Infect 2001; 127 (3): 461-7.

41.- Irwin D J, Millership S. Control of a community hepatitis A outbreak using hepatitits A vaccine. Commun Dis Public Health 1999; 2: 184-7.

42.- Prikazsky V, Oleár V, Cernoch A, Safary A, André F E. Interruption of an outbreak of hepatitis $A$ in two villages by vaccination. J Med Virol 1994; 44: 457-9.

43.- Zamir C, Rishpon S, Zamir D, Leventhal A, Rimon $\mathrm{N}$, Ben-Porath E. Control of a community-wide outbreak of hepatitis A by mass vaccination with inactivated hepatitis A vaccine. Eur J Microbiol Infect Dis 2001; 20 (3): 185-7.

44.- Marks P J, Fey R E, Parry J V, Deakin D, Carlisle D, Neal $R$ K. Use of hygiene advice and active immunisation to control an outbreak of hepatitis A. Commun Dis Public Health 2001; 4 (3): 158-62.

45.- Kaic B, Borcic B, Ljubicic M, Brkic I, Mihaljevic I. Hepatitis A control in a refugee camp by active immunization. Vaccine 2001; 19 (27): 3615-19.

46.- Iwarson S, Lindh M, Widerström L. Excellent booster response 4-6 y after a single primary dose of an inactivated hepatitis A vaccine. Scand J Infect Dis 2002; 34: 110-11.

47.- Landry P, Tremblay S, Darioli R, Genton B. Inactivated hepatitis A vaccine booster given > 24 months after the primary dose. Vaccine 2001; 19: 399-402.

48.- Beck B R, Hatz C, Bronnimann R, Herzog C. Successful booster antibody response up to 54 months after single primary vaccination with virosome-formulated, aluminum-free hepatitis A vaccine. Clin Infect Dis 2003; 37 (9): e126-8. Epub 2003 Sep 30.

49.- Williams J L, Bruden D A, Cagle H H et al. Hepatitis A vaccine: immunogenicity following administration of a delayed immunization schedule in infants, children and adults. Vaccine 2003; 21: 3208-11.

50.- Bryan J P, Henry C H, Hoffman A G et al. Randomized cross-over, controlled comparison of two inactivated hepatitis A vaccines. Vaccine 2001; 19: 743-50.

51.- Connor B A, Phair J, Sack D et al. Randomized, double blind study in health adults to assess the boosting effect of Vaqta or Havrix after a single dose of Havrix. Clin Infect Dis 2001.2 (1 February): 396-401.

52.- Zuckerman J N, Kirkpatrick C T, Huang M. Immunogenicity and reactogenicity of Avaxim (160AU) as compared with Havrix (1440 EL.U.) as a booster following primary immunization with Havrix (1440 EL.U.) against hepatitis A. J Travel Med 1998; 5: 18-22.

53.- Herzog C, Angst F, Beck B R et al. Boosting healthy travelers with a virosome-formulated hepatitis A vaccine after basic immunization with an alum-adsorbed hepatitis A vaccine. Abstracts of the $37^{\text {th }}$ Interscience Conference on Antimicrobial Agents and Chemotherapy, Sept 28-Oct 1, 1997. Toronto, Ontario, Canada. ASM Press. Abstr H-5, p 214.

54.- Black S, Shinefield H, Su L et al. Post marketing evaluation of inactivated hepatitis A vaccine (Vaqta, Merck) in 9740 children and adults. En Program and Abstracts of the $38^{\text {th }}$ Interscience Conference on Antimicrobial Agents and Chemotherapy, 1998. Washington DC, ASM Press. Abstr.

55.- Niu M T, Salive M, Krueger C et al. Two-year review of hepatitis A vaccine safety: data from the Vaccine
Adverse Event reporting System (VAERS). Clin Infect Dis 1998; 26: 1475-6.

56.- André F, Van Damme P, Safary A, Banatvala J. Inactivated hepatitis A vaccine: immunogenicity, efficacy, safety and review of official recommendations for use. Exp Rev Vaccines 2002; 1 (1): 9-23.

57.- Holzer B R, Hatz C H, Schmidt-Sissolak D, Glück R, Althaus B, Egger M. Immunogenicity and adverse effects of inactivated virosome versus alum-adsorbed hepatitis A vaccine: a randomized controlled trial. Vaccine 1996; 14 (10): 982-6.

58.- Fletcher M A, Hessel L, Plotkin S A. Human diploid cells strains viral vaccines. Biol Develop Stand 1998; 93: 97-107.

59.- Hess G, Clemens R, Bienzle U et al. Immunogenicity and safety of an inactivated hepatitis A vaccine in anti HIV-positive and negative homosexual men. J Med Virol 1995; 46; 40-2.

60.- Neilsen G A, Bodsworth N J, Watts N. Response to hepatitis A vaccination in human immunodeficiency virus-infected and uninfected homosexual men. J Infect Dis 1997; 176: 1064-7.

61.- Saglioca L et al. Efficacy of hepatitis A in prevention of secondary hepatitis A infection: a randomized trial. Lancet 1999; 353: 1136-9.

62.- Tregnaghi M. Control de hepatitis A en la Provincia de Córdoba, Argentina. Sesión del Comité Consultivo de Inmunizaciones, Sociedad Chilena de Infectología, mayo 2003. Datos no publicados.

63.- Craig A, Sockwell D, Schaffner W, Moore W, Skinner $\mathrm{J}$, et al. Use of hepatitis A vaccine in a communitywide outbreak of hepatitis A. Clin Infect Dis 1998; 27: $531-5$.

64.- D'Argenio P D, Adamo B, Cirrincione R, Gallo G. The role of vaccine in controlling hepatitis $A$ epidemics. Vaccine 2003; 21: 2246-9.

65.- Domínguez A, Salleras L, Carmona G, Batalla J. Effectiveness of a mass hepatitis A vaccination program in pre-adolescents. Vaccine 2003; 21: 698-701.

66.- Germinario C, Lopalco P L, Chirona M, Da Villa G, Chicanna M. From hepatitis B to hepatitis A and B prevention: the Puglia (Italy) experience. Vaccine 2000; 18: S83-5.

67.- American Academy of Pediatrics Committee on Infectious Diseases. Prevention of hepatitis A infections: Guidelines for use of hepatitis A vaccine and immune globulin. Pediatrics 1996; 98 (6): 1207 15.

68.- Advisory Committee on Immunization Practices ACIP. Prevention of hepatitis A through active or passive immunization. Recommendations of the Advisory Committee on Immunization Practices (ACIP). MMWR Morb Mortal Wkly Rep 1999, Oct 01. 48(RR12): 1-37.

69.- http://www.cdc.gov. Accedido 10 octubre 2003.

70.- American Academy of Pediatrics. Hepatitis A. En Pickering LK, ed. 2003 Redbook: Report of the Committee on Infectious Diseases $26^{\text {th }}$ ed. Elk Grove Village, Il.: American Academy of Pediatrics 2003:

71.- Crowcroft N S, Walsh B, Davison K L. Gungabissoon - V on behalf of PHLS Advisory Committee on Vaccination and Immunisation. Commun Dis Public Health 2001; 4 (3): 213-27.

Correspondencia a:

José Cofré Guerra

Email: pepecofre@123.cl 